\title{
Smooth Haptic Interaction in Broadcasted Augmented Reality
}

\author{
Jongeun Cha ${ }^{1}$, Beom-Chan Lee ${ }^{1}$, Jong-phil Kim ${ }^{1}$, Seungjun $\mathrm{Kim}^{2}$, and Jeha Ryu ${ }^{1}$ \\ ${ }^{1}$ Human-Machine-Computer Interface Lab., \\ \{gaecha, bclee, ryu\} @gist.ac.kr \\ Dept. Mechatronics, Gwangju institute of Science and Technology, Gwangju, Korea \\ ${ }^{2}$ System Integration Lab., \\ zizonedgist.ac.kr
}

\begin{abstract}
This paper presents smooth haptic interaction methods for an immersive and interactive broadcasting system combining haptics in augmented reality. When touching the broadcasted augmented virtual objects in the captured real scene, problems of force trembling and discontinuity occur due to static registration errors and slow marker pose update rate, respectively. In order to solve these problems, threshold and interpolation methods are proposed respectively. The resultant haptic interaction provides smoother continuous tremblefree force sensation.
\end{abstract}

\section{Introduction}

Rapid growth of computing and telecommunication technology allows recent digital multimedia systems to incorporate users' immersion and interactions. Physical touch to the digital multimedia may play an important role for immersion and interactions. O'Modhrain and Oakley [1] explored how haptic interaction might enhance and enrich the experience of broadcast contents. Moreover, much attention is newly given to the augmented reality (AR) technology [2] in broadcasting media production because of its simple but excellent interactive display and tracking potential. BBC [3] also introduced AR technology in broadcasting production. The haptic interaction in AR-based broadcasting system, however, may cause force trembling and discontinuity problems as observed in [4]. Virtual objects that are augmented in the captured scene showed trembling due to static registration errors. Moreover, touch to the moving objects generated abrupt change of force sensation because of the slow marker pose update rate compared to the high haptic rendering rate. In order to solve these problems, this paper presents smooth haptic interaction methods by threshold and interpolation techniques for haptically enhanced broadcast contents, which can be produced and broadcasted.

\section{Broadcasting System Chain with Haptics in Augmented Reality}

This section presents brief summary of the haptically enhanced broadcasting system that we proposed earlier [4] for explaining overview of the data flow. In Fig. 1, a captured video is analyzed in the AR process to detect fiducial markers in it in order 
to derive their 3D spatial relationship with respect to the camera coordinate system. ARToolKit [5] can facilitate this process. The 3D media database contains 3D object model information: geometry, texture, and even material properties for haptic sensation. In the proposed system, the 3D model data is downloaded to clients prior to video streaming because the $3 \mathrm{D}$ object model is not needed for every scene. Then the viewer can explore and manipulate the object at designated haptic interaction session that can be indicated on the screen by a producer. In the client site, the object is augmented to the scene and is displayed. Then haptic probe is overlaid on it. The control unit processes the video media and the 3D model data to control haptic device by using haptic rendering algorithm that is the main functions of haptic process. In our system, we have used the 6-dof haptic devices, Phantom [6].

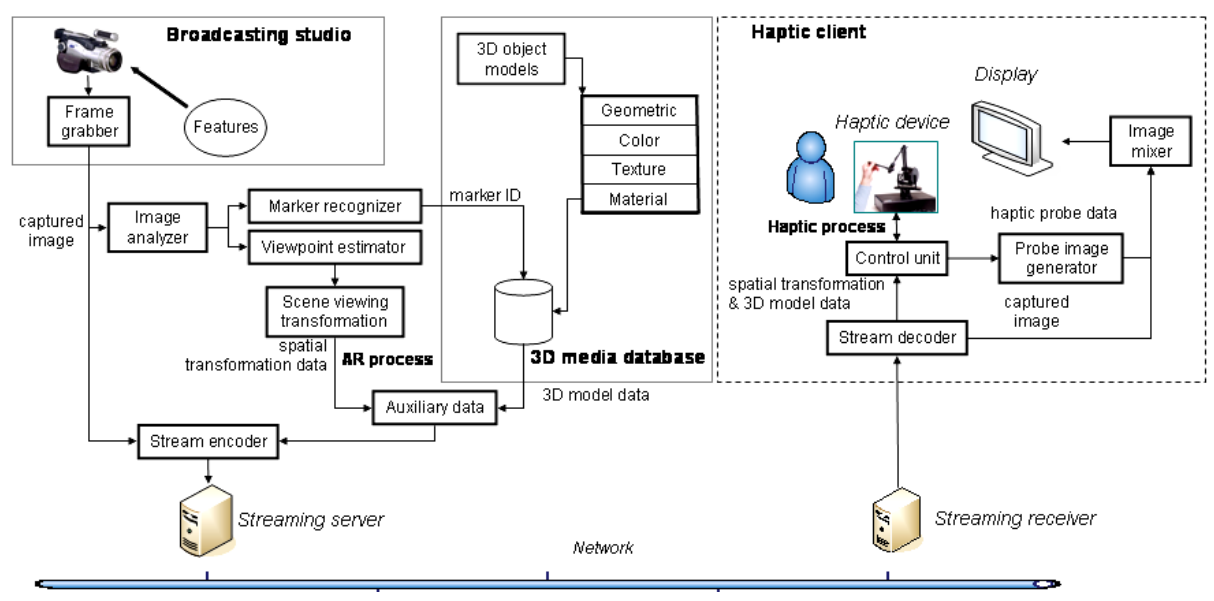

Fig. 1. The broadcast system chain

\section{Smooth Haptic Interaction in Augmented Haptics}

Implementation of the proposed haptically enhanced broadcasting system showed some abrupt and trembling force sensation to users. The first problem is that static registration errors occur from optical distortion, tracking errors, mechanical misalignments, and incorrect viewing parameters even when nothing is moving in the scene [2]. These errors lead to force trembling when viewers touch an augmented virtual object with a haptic interface. The estimated accuracy of ARToolKit for a single marker showed that the farther the marker distance from the camera and the smaller the tilt angle, the less the accuracy [7]. In order to estimate the maximum pose error to cut off, we measured the marker $(8 \times 8 \mathrm{~cm})$ pose fixed on the wall that is $70 \mathrm{~cm}$ distant from and perpendicular to the camera. Then we obtained the maximum static errors in the $70 \mathrm{~cm}$ camera workspace. Fig. 2 shows the results, where the left column indicates the $x-y-z$ position variations and the right column indicates roll-pitch-yaw angle variations. Then we have applied the threshold to almost completely eliminate the trembling based on the maximum pose variations given by equation in Fig. 2 . 


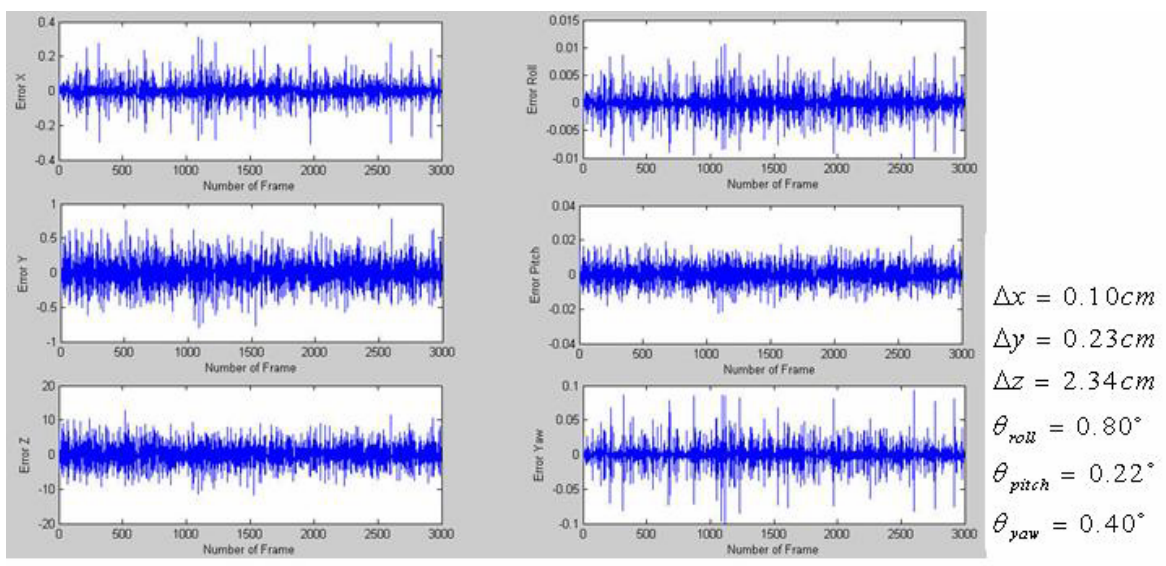

Fig. 2. Static pose variations

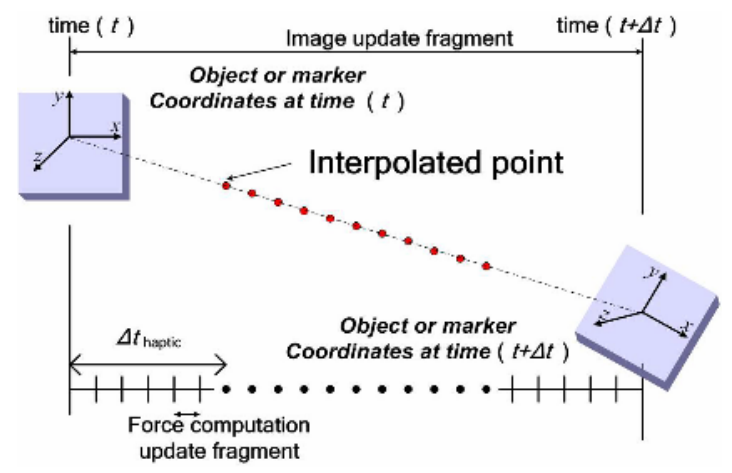

Fig. 3. Pose interpolation in haptic interaction

Humans can sense force vibrations well in excess of $300 \mathrm{~Hz}$. For smoother and more stable force feeling, generally, high haptic update rate $(>1000 \mathrm{~Hz})$ is required. But the estimated marker pose, that is the virtual object pose, is updated at around $20 \mathrm{~Hz}$, much slower than haptic update rate. This performance gap may cause the contact force discontinuous, that is to say, humans can perceive the discrete change of the virtual object. The haptic rendering loop calculates the interaction force in about $1 \mathrm{kHz}$ rate while the virtual object pose is updated in about $20 \mathrm{~Hz}$. In other words, between the two consecutive graphic rendering time $(t)$ and time $(t+\Delta t)$ in Fig. 3, the haptic rendering loop computes the interaction force about 50 times with the virtual object that is statically posed at time $(t)$. When the virtual object pose jumps to the next pose at time $(t+\Delta t)$, therefore, the viewer grabbing the haptic interface and contacting the virtual object feels uneven jumping force. Therefore, the virtual object pose must be interpolated between the poses at $(t)$ and $(t+\Delta t)$ in the haptic rendering loop. This interpolation is possible because when we display the augmented scene at $(t)$, we already have the virtual object poses at $(t)$ and $(t+\Delta t)$ because the streamed 
data is buffered in advance. Each interpolation pose in the haptic rendering loop between $(t)$ and $(t+\Delta t)$ was calculated by adding the pose at $(t)$ and the pose variation during $\left(\Delta t_{\text {haptic }}\right)$. The results indicate that the interpolation process is sufficient to give the apparent continuous force when the virtual object is moving.

\section{Conclusions and Future Works}

This paper presented smooth haptic interaction methods for an immersive and interactive broadcasting system combining haptics in augmented reality. Threshold and interpolation methods showed more stable and smoother force in haptic interaction. The estimated threshold values are a little sensitive to light condition and background scene. We will design and implement robust low-pass filter to eliminate the static registration errors in changing environments.

\section{Acknowledgement}

This work was supported by the Ministry of Information and Communication (MIC) through the Realistic Broadcasting IT Research Center (RBRC) at Gwangju Institute of Science and Technology (GIST).

\section{References}

1. S. O'Modhrain and I. Oakley,: TouchTV: Adding Feeling to Broadcast media, Proc. the $1^{\text {st }}$ European Conf. Interactive Television: from Viewer to Actors, Dec. (2003) 41-47

2. R. Azuma,: A survey of augmented reality, Presence : Teleoperators and Virtual Environments, Vol. 6, No. 4 (1997) 355-385

3. A. Woolard, V. Lalioti, N. Hedley, N. Carrigan, M. Hammond and J. Julien,: Case Studies in Application of Augmented Reality in Future Media Production, Proc. the Second IEEE and ACM Int. Symp. Mixed and Augmented Reality, Oct. (2003)

4. J. Cha, J. Ryu, S. Kim, S. Eom and B. Ahn,: Haptic Interaction in Realistic Multimedia Broadcasting, Proc. 5th Pacific Rim Conf. Multimedia on Advances in Multimedia Information Processing, Part III, Nov./Dec. (2004) 482-490

5. ARToolKit, http://www.hitl.washington.edu/artoolkit

6. Phantom, SensAble Technologies, http://www.sensable.com

7. D. F. Abawi and J. Bienwald,: Accuracy in Optical Tracking with Fiducial Markers: An Accuracy Function for ARToolKit, Proc. the Third IEEE and ACM Int. Symp. Mixed and Augmented Reality, Nov. (2004) 